\title{
Accelerated Recovery Through Ultrasound Therapy: Lactic Acid Clearance and Muscle Recovery
}

\author{
David 0 Draper* \\ Brigham Young University, USA
}

*Corresponding author : David O Draper, Professor of athletic training and sports medicine, Brigham Young University, Provo, Utah, USA.

Received Date: December 14, 2018

Published Date: December 17, 2018

\section{Sustained Acoustic Medicine}

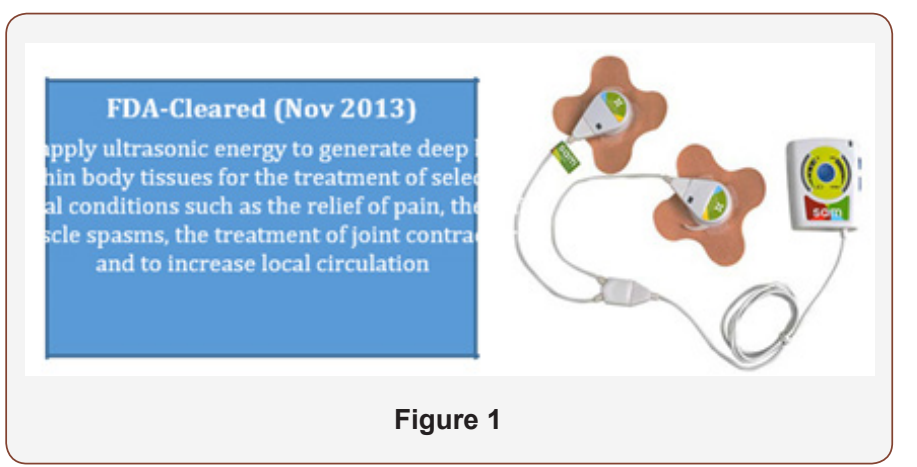

sam ${ }^{\circledR}$ Professional System (Portable, wearable, low intensity therapeutic ultrasound device - Patient self-administers treatment for up to 4 h per day)

What is sam?

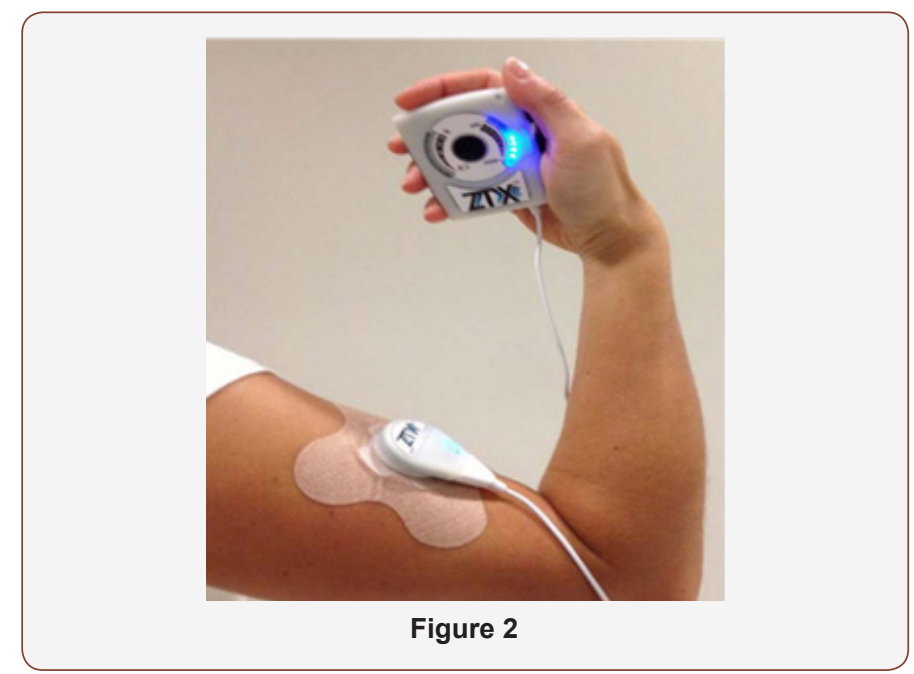

- $\quad$ Long duration - up to 4 hours

- $\quad$ Low intensity- $0.132 \mathrm{~W} / \mathrm{cm}^{2}$

- Duty Cycle- $100 \%$
It is possible to deliver these long duration low Intensity treatments because the device is:

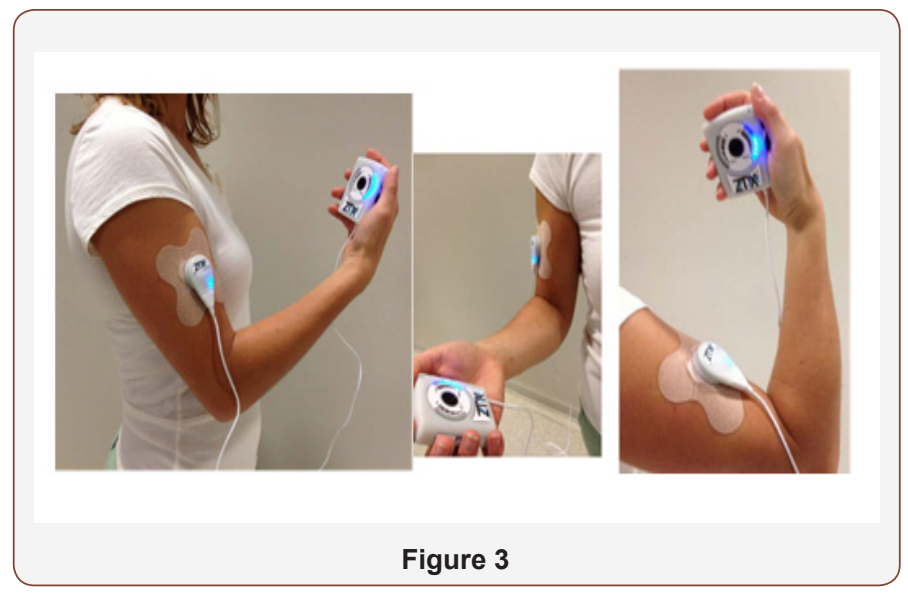

- $\quad$ Portable

- $\quad$ Small (about the size of a TENS unit

- Battery-Operated

- $\quad$ Self-administered

- Comfortable

- Worn during the course of daily life

ZetrOZ - Long Duration Low Intensity Therapeutic Ultrasound Device

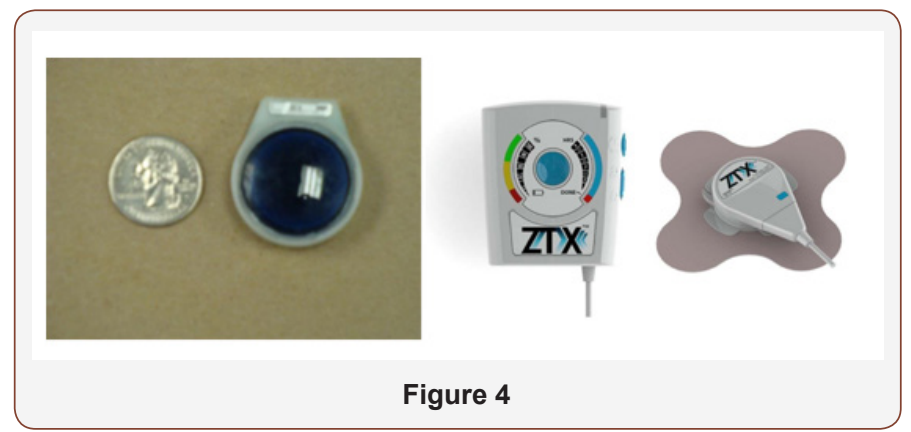




\section{Indicated for:}

- Relief of Pain
- Relief of Muscle Spasms

- Local Increase in Circulation

\section{Schedule of Assessments}
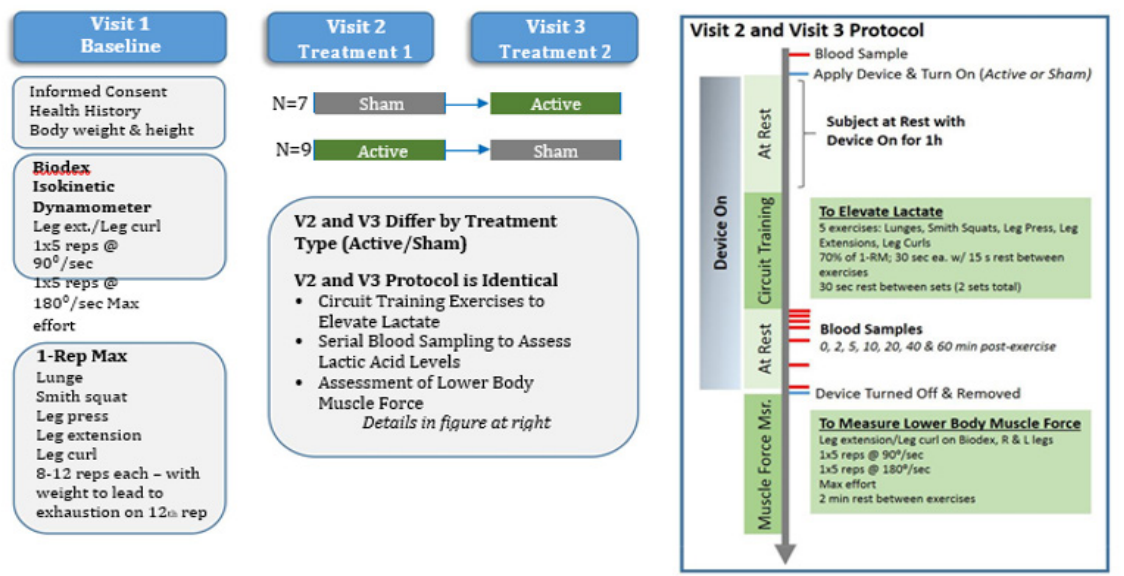

Figure 5

\section{Past Research}
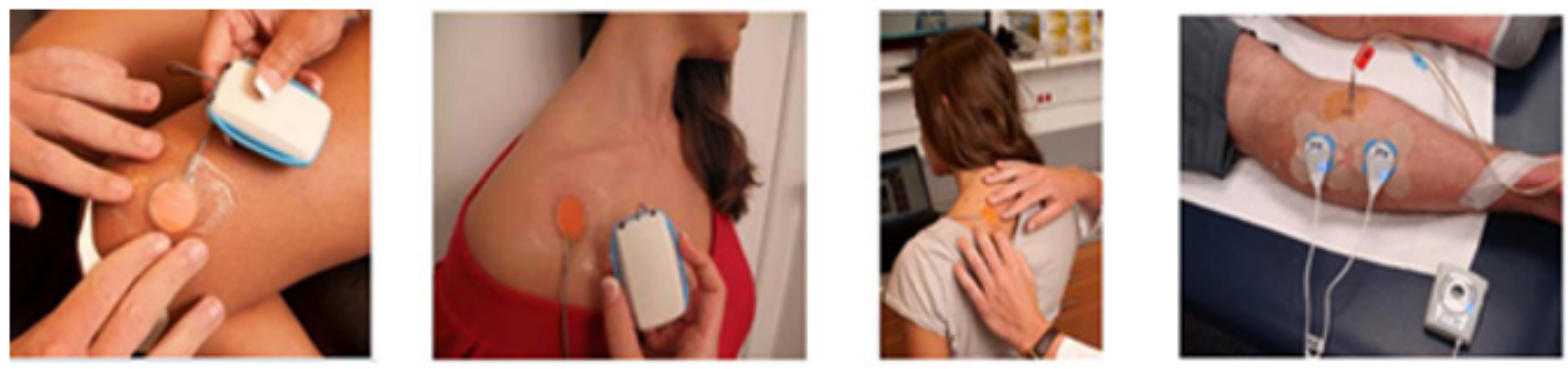

Figure 6

- $\quad$ Studies on knee osteoarthritis, shoulder tendinitis, and trapezius muscle spasm have all found the SAM to be effective in substantially decreasing pain

- A study at BYU found that the SAM produces moderate to vigorous heating in human muscle

Placement of Applicators on Study Subjects

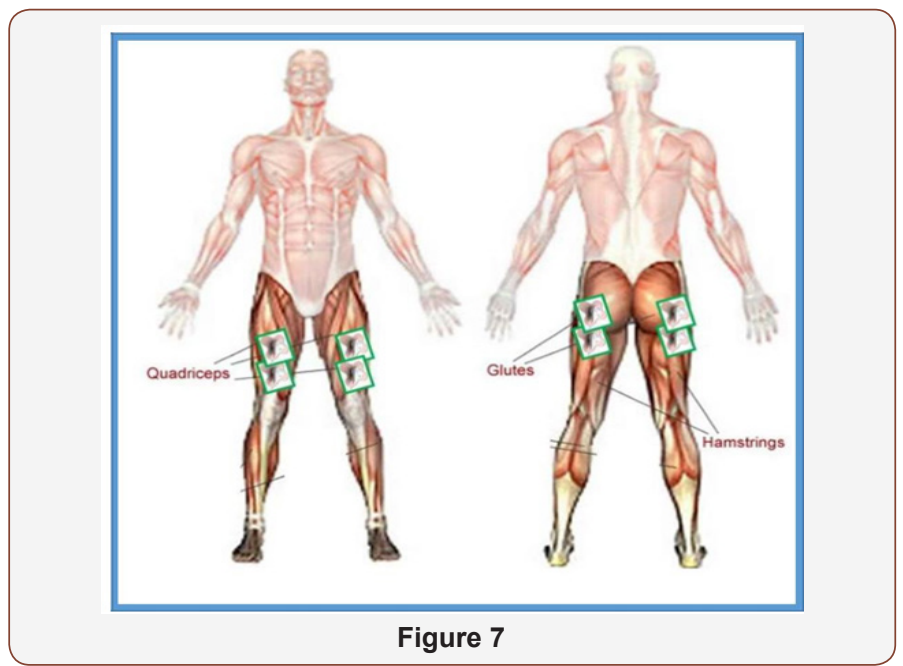

For each Subject

- 4 Power Controllers

- 8 Applicators

- $\quad$ Power Controller per 2 Applicators

Placement of Applicators

- $\quad$ Applied to L and R legs

- 2 applicators each on quadriceps and hamstrings/glutes as shown in figure

\section{Ultrasound Benefits:}

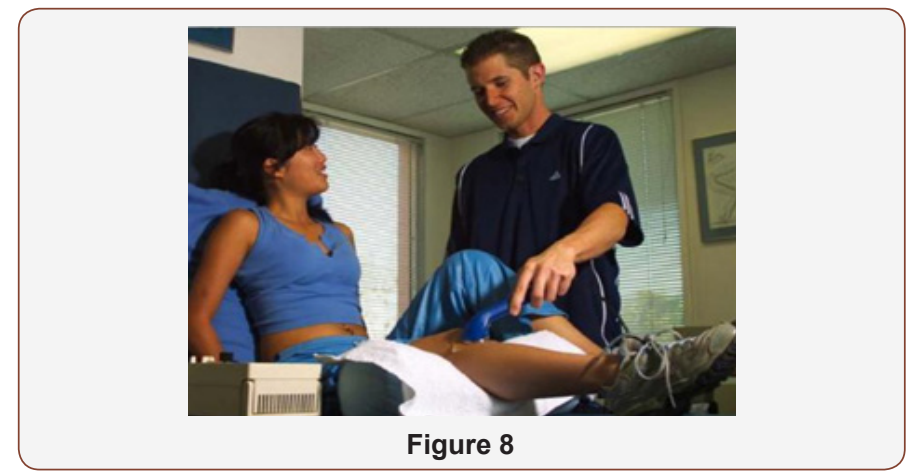


- $\quad$ Reduced pain

- Reduced inflammation

- Increased healing rate

- $\quad$ Non-invasive

- $\quad$ Simple treatment

\section{Traditional:}

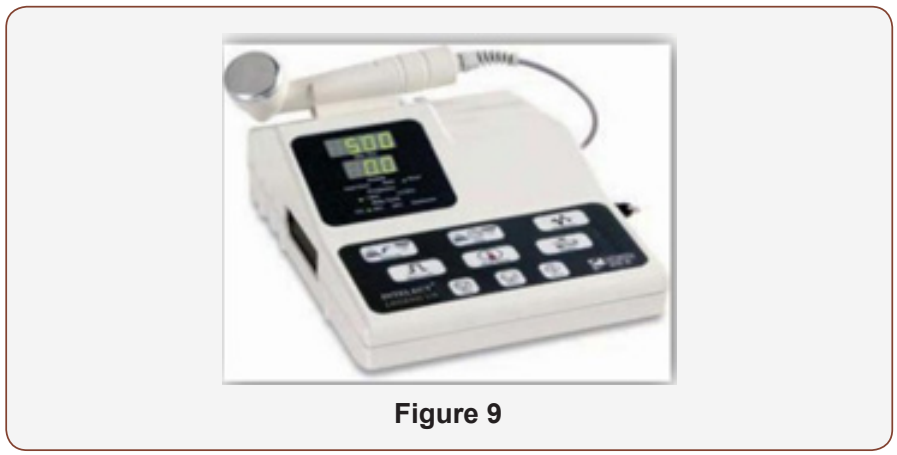

- $\quad$ Large size and cost

- Not portable

- $\quad$ Treatment time 5-15 mins

- $\quad$ Applied by clinician

- Messy gel

\section{Wearable Solution:}

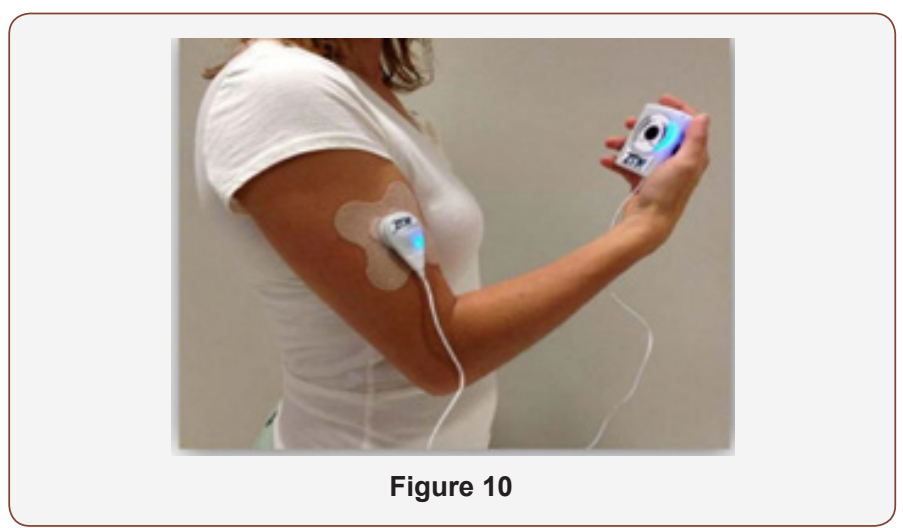

- Wearable

- $\quad$ No Mess

- Use Daily

- $\quad$ Long Lasting

- Battery Powered

\section{Rationale}

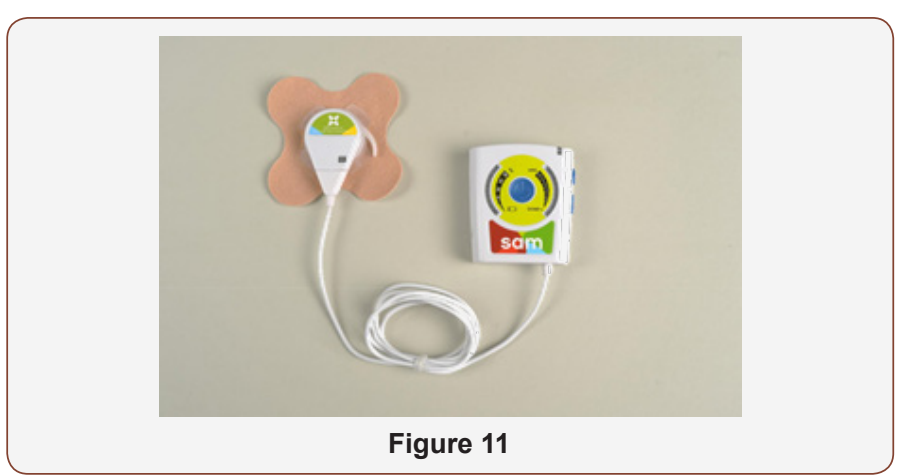

- Muscles become fatigued during intense exercise

- Athletic performance and training will be enhanced if muscle fatigue occurred slower and muscle recovery occurred faster

- Therapeutic ultrasound has been associated with increased local blood flow1, which may enable muscles to perform for longer periods of time or recover faster after exercise

\section{Study 1: Objective \& Design}

\section{Study Objective}

- Determine if wearing a long-duration ultrasound therapy device on muscles can accelerate lactic acid kinetics to promote clearance from blood

\section{Study Design}

- Double-blind, randomized, cross-over design

- Healthy volunteers: male subjects currently participating in resistance training 3-5 days/week for $\geq 3$ months

- $\quad$ Site: SUNY Brockport, Department of Exercise Science and Athletic Training

SAM Treatment Accelerated Clearance of ExerciseInduced Blood Lactate Accumulation

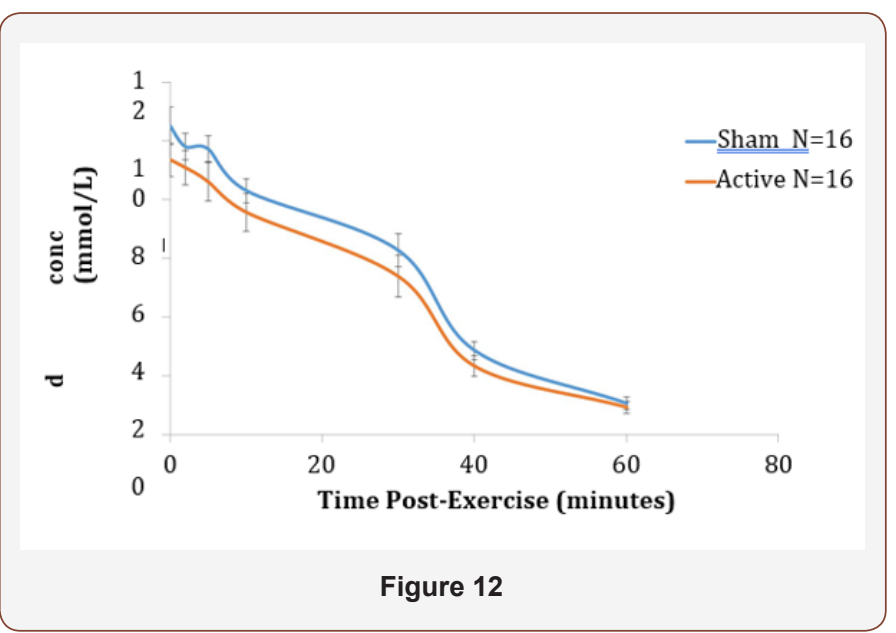

Post-exercise lactate concentrations were adjusted by the pre-exerciseconcentration to reflect clearance of exercise-induced lactate

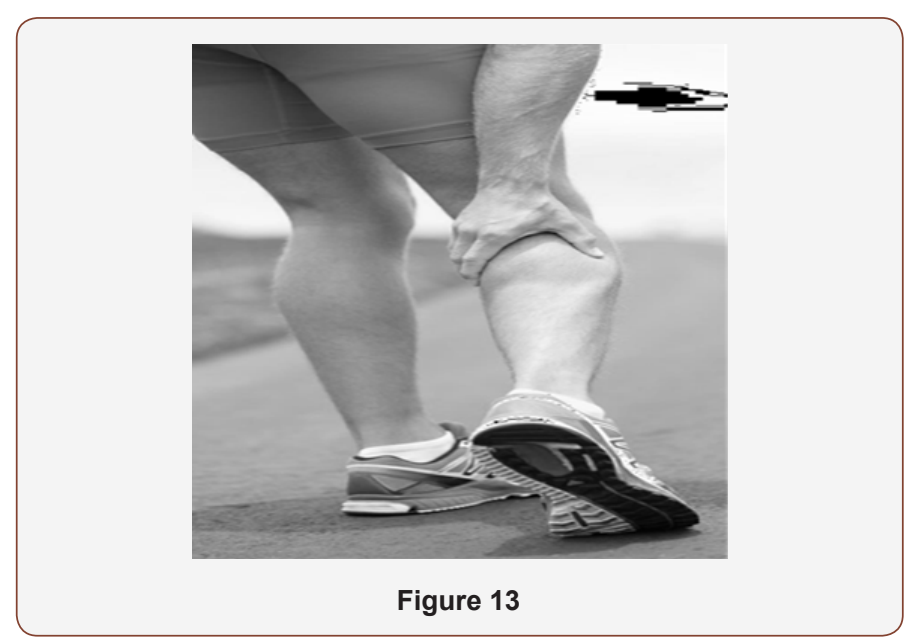




\section{Study 1: Objective \& Hypothesis}

\section{Objective}

- $\quad$ Lactic acid (and its conjugate ion, lactate) are produced during exercise through the reduction of pyruvate

- Lactic acid/lactate accumulates in muscle during exercise, contributing to acidosis and exhaustion can be removed by several techniques including ultrasound therapy, massage, stretching, and passive recovery

- $\quad$ Output Measure: Blood Lactate levels

- $\quad$ Correlated with return to pre-exercise conditions
- $\quad$ Can be measured through a readily available blood test

\section{Hypothesis}

- Ultrasound therapy accelerates lactic acid clearance postexercise.

\section{Quantifying Lactate Exposure Area Under the Curve (AUC)}

- Area under blood lactate curve corresponds with the total dose over time in the body

- Used for analysis, crossover design permits use of a paired t-test to compare values

\begin{tabular}{|c|c|c|}
\hline Subject & AUC (Pre Ex) Placebo & AUC (Pre Ex) Active \\
\hline 1 & 214.91 & 516.93 \\
\hline 2 & 510.615 & 329.51 \\
\hline 3 & 293.96 & 392.9 \\
\hline 4 & 371.545 & 428.755 \\
\hline 5 & 389.93 & 269.435 \\
\hline 6 & 251.695 & 220.105 \\
\hline 7 & 190.28 & 152.51 \\
\hline 8 & 292.395 & 302.41 \\
\hline 9 & 262.965 & 172.685 \\
\hline 10 & 241.905 & 263.465 \\
\hline 11 & 393.8125 & 144.2225 \\
\hline 12 & 236.0275 & 277.055 \\
\hline 13 & 351.0525 & 187.1225 \\
\hline 14 & 254.1625 & 172.79 \\
\hline 15 & 270.905 & 196.185 \\
\hline 16 & 268.93 & \\
\hline
\end{tabular}

Average $13 \%$ decrease in total AUC over $1 \mathrm{~h}(\mathrm{p}=0.0287)$

\section{Muscle Force Results}

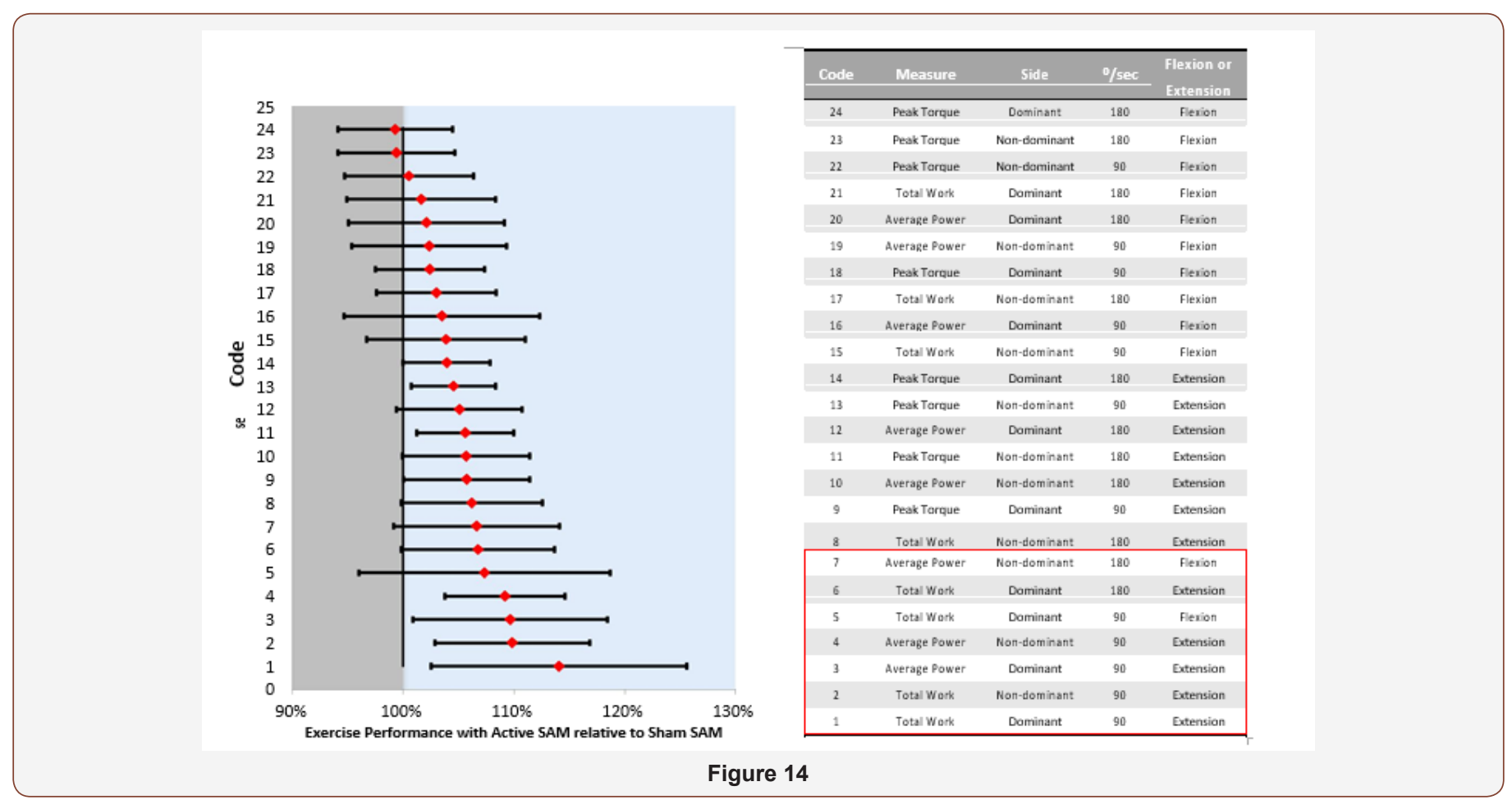




\section{Study 1 Summary}

\section{Blood Lactate Clearance}

- $\quad$ Treatment with Sustained Acoustic Medicine resulted in accelerated clearance of blood lactate within 1-h post-exercise

\section{Muscle Force}

- $\quad$ Significantly greater muscle force exerted with Active v Sham SAM as measured by Total Work and Average Power

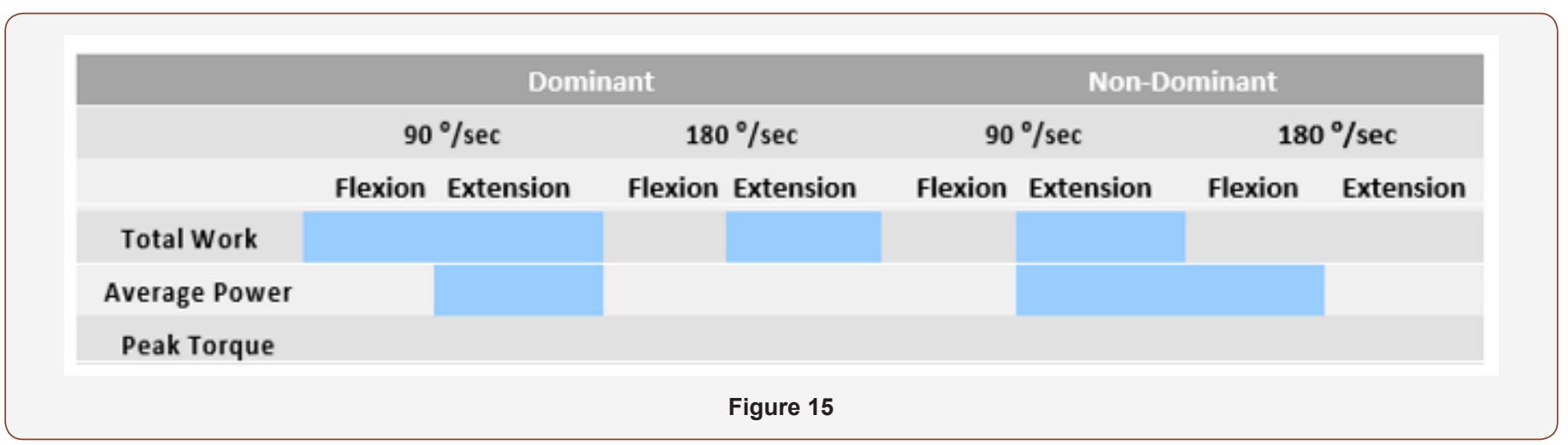

Significant differences between Active and Sham indicated with blue shading

\section{Study 2: Objective \& Hypothesis}

\section{Study Objective}

- Determine if wearing a long-duration ultrasound therapy device on muscles can aid post-exercise muscle recovery and thereby positively impact subsequent athletic performance among professional cyclists

\section{Hypothesis}

- If cyclists perform physical exertion and then wear the ZetrOZ device on the main muscles that are activated during cycling (i.e. quadriceps, hamstrings, and gluteus maximus), then the muscles will recover faster because ultrasound therapy speeds nutrient transport and increases circulation, both of which are involved in muscle recovery. Faster recovery will be reflected by less of a decrease in power output in a subsequent workout among subjects treated with the device.

\section{Study Design}

1. Double-blind, randomized, cross-over design

2. Study Population

- Professional Cyclists with race-training experience, experience with power meters

- 8 males, 4 females

- 25-45 years

3. Same applicator placement as Study 1 (4 applicators per leg)

4. Outcomes

- Power (W) and total work (KJ) during each 5 minute maximum effort

- $\quad$ Pain (NRS), Perceived Improvement (GROC) in Daily Diary

5. Site: Legaci Cycling Group, Rochester, NY (T Scheske)

\section{Study Protocol}

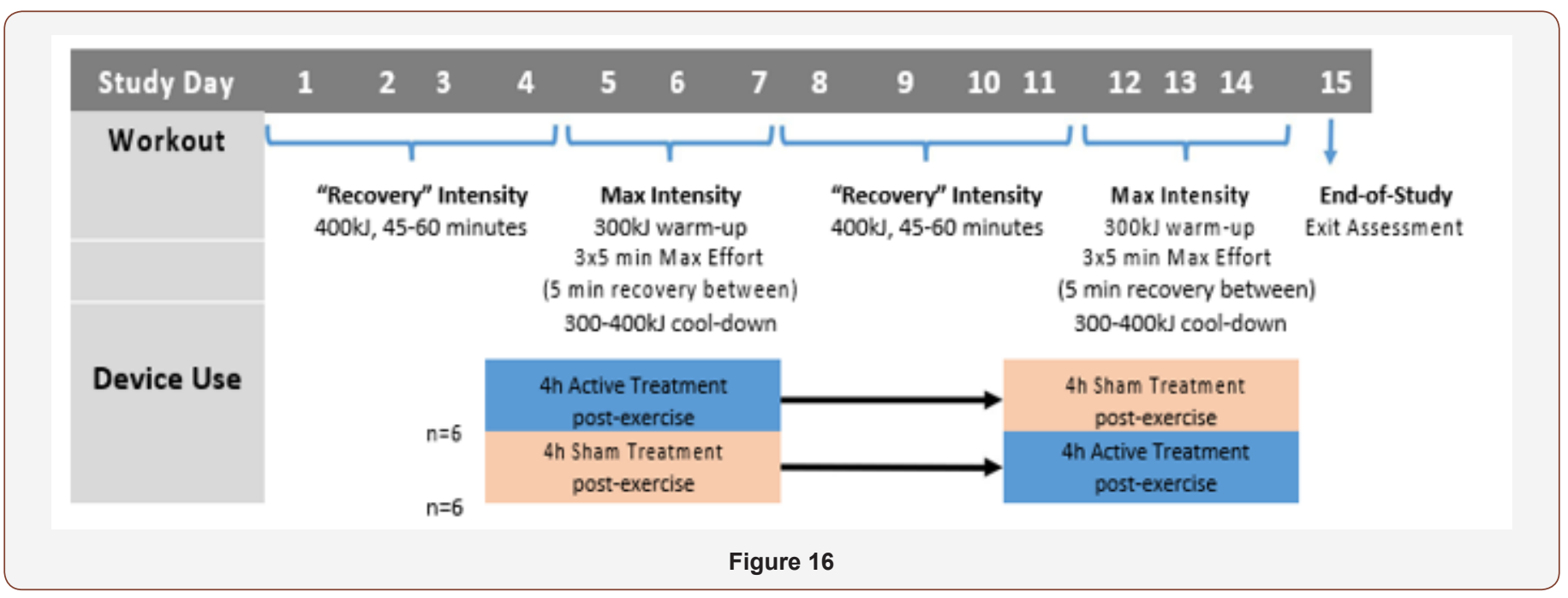

\section{Cyclist Study Preliminary Results}

\section{Study Status}

- $\quad$ All subjects enrolled and completed, analyses underway
2. Subjects exhibited greater energy output during 6 of 9 5-min Max Effort sessions with the active device than the sham device 
3. On Day 3 of the Max Intensity workouts, riders averaged $1 \%$ more work per 5 minute sprint with active treatment than sham

- Approximately equivalent to riding 50 additional feet over 5 minutes

4. Results of pilot study indicate larger and more extensive study could be conducted to meaningfully examine impact of SAM on muscle recovery

\section{Conclusions and Future Work}

\section{Conclusions}

- $\quad$ SAM Therapy significantly accelerated post-exercise lactate recovery

- $\quad$ SAM Therapy facilitated increased muscle force after a taxing exercise regimen

- $\quad$ SAM Therapy has been piloted to prevent fatigue and maintain muscle performance over a multi-day training regimen in professional cyclists

\section{Future Research}

- Investigate when device use provides greatest benefit on lactate clearance (before, during, or after exercise)

- Study impact of SAM on muscle performance in different types of exercise, different kinds of athletes

- Investigate how long muscle performance is extended following treatment

- $\quad$ Conduct fully powered study to assess impact of SAM on muscle recovery in professional athletes

\section{Acknowledgements}

1. Study Funded by: ZetrOZ, Inc.

2. ZetrOZ, Inc. is the manufacturer of sam $\AA$.

\section{Conflict of Interest}

None. 